\title{
The Impact of Persistent Current Field Frrors on the Stability of the Proton Beam in the HERA Proton Ring
}

\author{
F. Willeke and F. Zimmermann \\ DESY. Notkestr. 85, 2000 Hamburg 52, Germany
}

\section{INTRODUCTION}

With the start of commissioning of the HERA proton ring, a second large superconducting storage ring has come into operation. HERA-p differs from the first superconducting circular accelerator, the TEVATRON, by its low injection energy which is with $\mathrm{E}_{\text {inj }}=40 \mathrm{GeV}$ more than 20 times smaller than the top energy of $E=820 \mathrm{GeV}$. Furthermore the requirements on the stability of the beam at injection energy are in the case of the HERA proton ring much higher than for the other large proton accelerators. The rate of acceleration is considerably lower than for the TEVATRON or the SPS, and since the filling procedure takes about $15-30 \mathrm{~min}$. HERA-p has to be operated as a storage ring at injection energy.

The field in superconducting magnets at low excitations is strongly perturbed by persistent currents (p.c.) which are eddy currents in the superconductor. Conventional room temperature magnets can be designed so that the nonlinear field errors do not exceed the sextupolar nonlinearities which are introduced intentionally in the machine to compensate the chromaticity. At injection the nonlinear field errors in the magnets of the HERA proton ring exceed this natural amount of nonlinearity by a factor of five. Persist $n t$ current driven field errors are not only sextupolar bu1 also include higher order multipolar components which have dipole svmmetry. Therefore, large superconducting arcelerators like HERA-p need a multipole field correction susiem to provide suitable conditions for proton storage. Strong efforts have been made to design an approprale correction system. The motion of the protons in the machine has been extensively studied by particle tracking. Nen methods of analyzing the simulation data have been developed in order to ensure that the correction svstem provides sufficient stability.

A further complication arises from the fact that the persistent currents are subject to a slow logarithmic decay in time. There is a lot of experimental evidence from TEVATRON operation [1] and from systematic measurements on the HERA superconducting magnets. This decay depends in a complicated way on magnet history and the peak excitation $[4]$. Moreover, at the start of acceleration, the full strength of field errors is quickly reinduced. This requires complicated, programmed steering of the correc- tions or on-line magnetic field measurements.

This report starts with a short summary of the field errors of the HERA magnets under the special aspect of the stability of the proton beam. The dynamics of the proton beam under the influence of strong persistent field errors and the correction and compensation scheme of HERA is presented and discussed. The report closes with first results from the commissioning of the HERA proton ring

\section{MAGNET MEASUREMENTS}

Persistent currents are eddy currents in the filanents of the superconducting cable which are induced by the change of flux density due to the ramp of the main field (or any other magnetic field, the coil of the magnets is exposed to). The corresponding magnetization reaches a saturation value if the sum of eddy currents and transport current density reaches the critical current density $J_{c}$ which depends on properties of the superconductor, the temperature of the coil and the strength of the magnetic field around the filaments. The magnetization which always acts against a change in the magnetic field rearhes its maximum strength early in the magnet's excitation cycle. At high excitation. the transport current has driven the eddy current out of the cable and p.c. effects become negligible. On the ramp down. the eddy currents are induced with opposite sign. Since the magnetization is proportional to the local magnetic induction which is not uniformly distributed over the coil. the p.c.'s cause not only an attenuation of the main field but also a field distortion with strong sextupole and decapole components in the case of dipole magnets and strong 12-pole components in quadrupole magnets. In the case of the HERA magnet. the p.c. sextupole and decapole components reach their maximum value close to the injection field. According to ref $[2$, the average value of the p.c. sextupole component $b_{3}$ is then as large as $\left\langle\mathrm{b}_{3}\right\rangle=-33 \cdot 10^{-4} \mathrm{a}_{\mathrm{r}}=25 \mathrm{~mm}$ and the average decapole is $\left\langle b_{5}\right\rangle=+12 \cdot 10^{-4}$. The quadrupole field is distorted by a large 12-pole $\left\langle b_{6}\right\rangle=.22 \cdot 10^{-4} \hat{a}_{\mathrm{r}}=25 \mathrm{~mm}$. 


\begin{tabular}{|c|c|c|c|c|}
\hline \multicolumn{5}{|c|}{ TableI: Results of HERA Magnet Measurements } \\
\hline \multicolumn{5}{|c|}{ Multipole Coefficients Iow Field (40GeV) } \\
\hline & \multicolumn{2}{|c|}{ Normal Component } & \multicolumn{2}{|c|}{ Skew Component } \\
\hline order & average & sigma & average & sigma \\
\hline 1 & - & - & $\cdots$ & - \\
\hline 2 & 0.01 & 0.61 & -0.17 & 2.53 \\
\hline 3 & -32.47 & 3.24 & -0.36 & 0.71 \\
\hline 4 & 0.09 & 0.36 & 0.86 & 1.25 \\
\hline 5 & 12.39 & 1.04 & $(1.23$ & 0.66 \\
\hline 6 & -0.12 & 0.34 & -0.70 & 0.56 \\
\hline T & -2.17 & 0.53 & -0.20 & 0.54 \\
\hline 8 & 0.14 & 0.41 & 0.24 & 0.39 \\
\hline 9 & 0.46 & 0.48 & 0.10 & 0.67 \\
\hline \multicolumn{5}{|c|}{ Multipole Coefficients High Field (820GeV) } \\
\hline & \multicolumn{2}{|c|}{ Normal Componen 1} & \multicolumn{2}{|c|}{ Skew Component } \\
\hline order & average & sigma & average & sigma \\
\hline 1 & - & 18.00 & - & - \\
\hline 2 & -0.02 & 0.59 & -0.25 & 1.82 \\
\hline 3 & 0.10 & 2.70 & -0.25 & 0.39 \\
\hline 4 & 0.12 & 0.24 & 0.30 & 0.94 \\
\hline 5 & 1.12 & 0.78 & -0.05 & 0.18 \\
\hline 6 & 0.04 & 0.10 & -0.09 & 0.22 \\
\hline 7 & 0.21 & 0.27 & -0.01 & 0.10 \\
\hline 8 & 0.02 & 0.07 & 0.01 & 0.12 \\
\hline 9 & -0.37 & 0.12 & 0.01 & 0.09 \\
\hline
\end{tabular}

A remarkable feature of these field errors. which is very important for the beam stability. is that they are systematic. This means that they do not vary much from magnet to magnet. Therefore the spread of $b_{3}$ and $b_{5}$ values for the magnets at low excitation is not much larger than the high excitation spread in $b_{3}$ and $b_{5}$ due to geometric imperfections of the coil. This important fact is demonstrated in Fig 1 where the $b_{3}$ values for low excitation are plotted against the corresponding values at high excitation for 286 dipole magnets

HERA superconducting magnets have been manufactured in two production lines which vield systematic differences in the values of integrated field $\left(\Delta<\int \mathrm{Bdl}>=0.18 \%\right.$ ) and in the sextupole component $\left.\Delta, b_{3}>=35 \cdot 10^{-4}\right)$. The magnets of the two manufacturers are therefore installed in different quadrants of the machine. This allows a vendor-specific adustment of dipolar and sextupolar corrertors.

The p.c.'s in the superconducting cable of the HERA magnets decay slowly with time. This behaviour is believed to be due to flux creep as firsi described by Anderson 3 . The complex behaviour however which is observed in the HERA magnets cannot be fully explained by this model. It is well known that the decay of persistent currents depends on small variations of properties of the superconductor, caused for example by impurities, manufacturing techniques or mechanical stresses. This might be the reason why in the case of HERA, the time dependence of $b_{1}$ and $b_{3}$ not only varies significantly between the magnets of the two production lines by a factor of two but that there is also a considerable spread in the change of $b_{3}$. $\Delta \mathrm{b}_{3}^{\mathrm{rms}}(\Delta t=1800 \mathrm{~s})=2 \cdot 10^{-4}$ in the magnets from one vendor [4]. Thus during the long stored beam operation at 'flat-bottom' in HERA one has two sources of nonsystematic nonlinear field errors, geometric imperfections and a spread in p.c. time decay. The resulting spread is however not very large and turns out to be smaller than the values which have been obtained for the TEVATRON magnets 5 ?. There is however. one exception. HERA dipole magnets exhibit an unusully strong skew quadrupole component which has a spread of $<a_{2}^{r m}>=2.5 \cdot 10^{-4}$. The reason for this skew quadrupole component is not fully understood. The as change strongly with excitation but do not show the hysteresis of persistent current driven field errors. A possible reason is an asymmetric distribution of mechanical pressure. Table I summarizes the field quality of the HERA-p superconducting magnets

\section{BEAM DYNAMICS}

The high value of the systematic persistent current sextupole in the HERA-p dipole magnet at injection energy requires a non-lumped, distributed correction. This led to the concept of a $6 \mathrm{~m}$ long correction coil which is wound on the beam pipe inside the $9 \mathrm{~m}$ long dipole magnet.

Each half FODO cell has two dipoles. The sextupole correction coils are located at the quadrupole end of the dipoles so that these correction coils call also be used for chromaticity correction. This isa a mpromise between local sextupole correction and effective chromaticity compensation. A comparison of this arrangement with a situation without strong systematic sextupole error and with lumped chromaticity correctors near the quadrupoles gives only marginal differences for the beam stability.

Beam stability is checked by particle tracking based on the computer code RACETRACK $[1]$. As a figure of merit we use the dynamic aperture which is the domain around the closed orbit inside which the particles perform stable oscillations. Only single particles are considered in the model. No other effect than magnet nonlinearities are taken into account for calculation of dynamic aperture. This property is defined as the maximum oscillation amplitude which exhibits regular, non-chaotic behaviour. This is checked by the Lyapunov exponent method $[6,7]$

From the higher order multipole components of the HERAp dipole field, it is only the decapole which is important for the dynamic aperture. The impact of the systematic persistent current driven decapole is, besides its unusual strength of $b_{5} \sim 12 \cdot 10^{-4} a_{r}=25 \mathrm{~mm}$, the same as for any other field error with dipole symmetry. Since the lattice and beam optics is very regular in the arcs. the excitation of nonlinear resonances and nonlinear phase space distortions is small due to intrinsic cancellation. Therefort particles with the design momentum do not suffer much from systematic field errors which are symmerric around par- 
ticle orbit. The damage, however, becomes apparent if off-momentum particles are considered. Since the central orbit of the particles is displaced with respect to the center of the magnet. the particle also experiences antisymmetric forces which give rise to an amplitude dependent tune shift or detuning which always builds up over the lattice. The detuning causes a reduction of the stable amplitudes. The mechanism is well-known and has been discovered by Chirikov [9]. The amplitude dependent tune shift causes an overlap of the nonlinear resonances. Then the nearby closed surfaces in phase space which confine the motion of the particles are destroyed. Particles can diffuse slowly through phase space until ther eventualis reach domains with strong nonlinear forces which cause a rapid growth in amplitude and a loss occurs.

The simulations of the dynamic aperture in HERA-p in presence of p.c. field errors as a function of a constant momentum deviation show a typical behaviour. The $d y$ namic aperture decreases almost linearly with increasing momentum deviations (see Fig. 2, see also ref 10 . For HERA, the corresponding reduction of dynamic aperture was considered intolerable. Thus a decapole correction was needed.

An effective correction of the damage caused by the persistent current decapole has to concentrate on the detuning for off-momentum particles. The optimum location for a single corrector per half cell is the middle of the half cell. The available $1 / 3$ of one of the two dipole magnets was used for a $3 \mathrm{~m}$ long beam pipe wound decapole correction coil. The tune shift for oscillation amplitudes of $27.6 \mathrm{~mm}$ and a $\Delta p / p=2^{\circ} / 0$ amounts to $\Delta Q \sim-0.02$. In order to compensate this tune shift the correction coil needs to be excited to 75 gauss $\mathrm{m} / \mathrm{a}=25 \mathrm{~mm}$. Fig. 3 shows how the dynamic aperture for a particle with $\Delta \mathrm{p} / \mathrm{p}=2^{\mathrm{c}} / \mathrm{co}$ recovers as a function of corrector strength (in units of integrated p.c. decapole per half cell). For optimum corrector setting of $\equiv 100 \%$ one obtains the dynamic aperture versus constant momentum offset as given by the $(\Delta)$ in Fig. 2.

The impact of the p.c. 12-pole of the quadrupole magnets is even more dramatic. Since the antisymmetric dodecapole field produces a detuning term independent of momentum, the dynamic aperture is also reduced for onmomemtum particles by more than a factor of $3[10$. The dodecapole amounts to $-22 \cdot 10^{-4}(\mathrm{r}=25 \mathrm{~m}=\mathrm{m}$ which causes a tune shift $\left(\bar{a}_{\mathbf{r}}=27 \mathrm{~mm}\right)$ of $\Delta Q_{x} \sim 0.15$. A dodecapole correction coil in each quadrupole has been installed to compensate this effect.

Essential for good dynamic aperture in HERA is the compensation of the coupling resonance which is excited by the strong skew quadrupole components in the HERA dipole (see also 13 ). For compensated coupling the optimum tunes are found to be $Q_{x}=31.15$ and $Q_{y}=32.18$ close to the main tune diagonal. For uncompensated coupling, the dynamic aperture is reduced by almost $20 \%$. Simulation of stability in the HERA proton ring using the

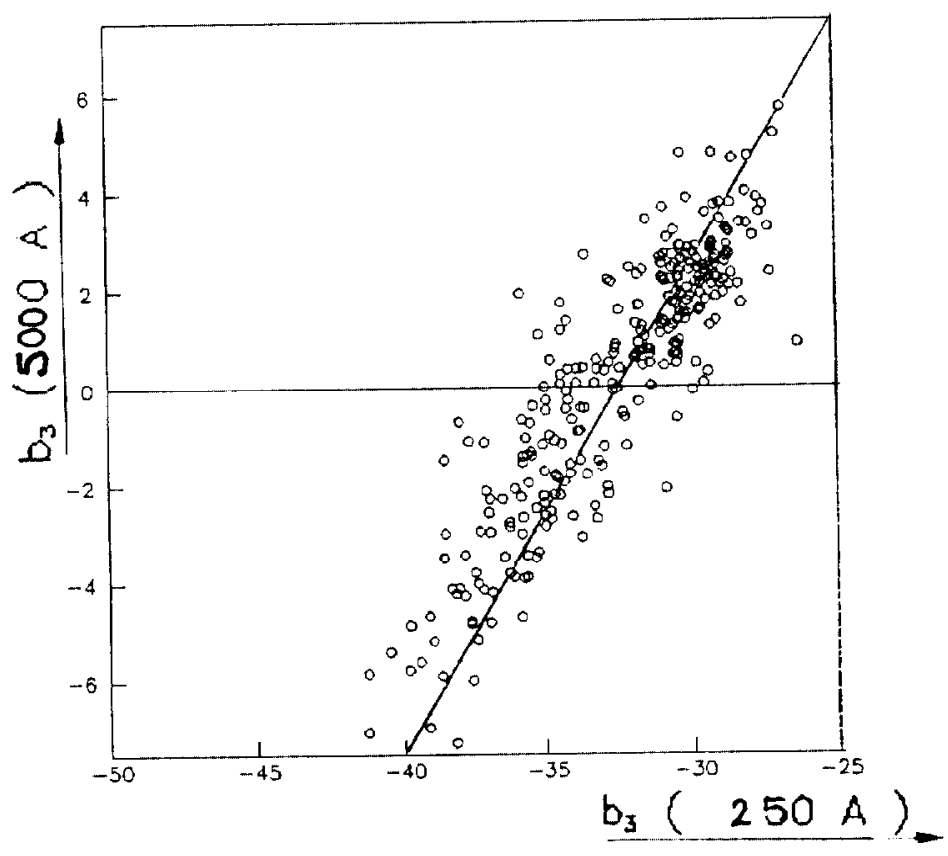

Figure 1: Low excitation Sextupole Components vs High Excitation Sextupole Fields of the HERA superconducting Dipole Magnets

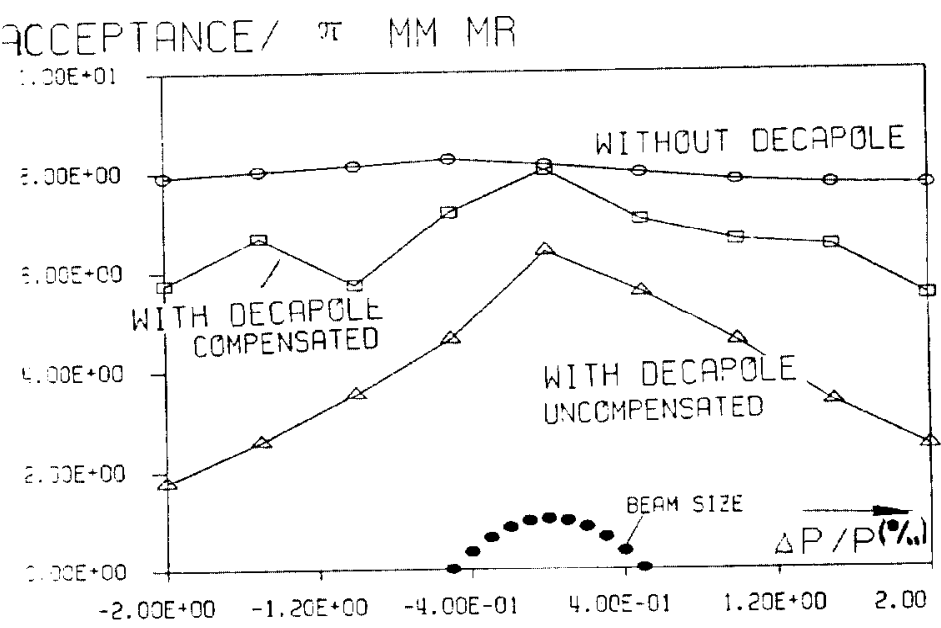

Figure 2: Acceptance of the HERA proton Ring is. Constant Momentum deviation. Circles: simulation results with no decapole. Triangles: with $b_{3}=12 \cdot 10^{-4}$ and no compensation. Squares: with decapole and compensation 


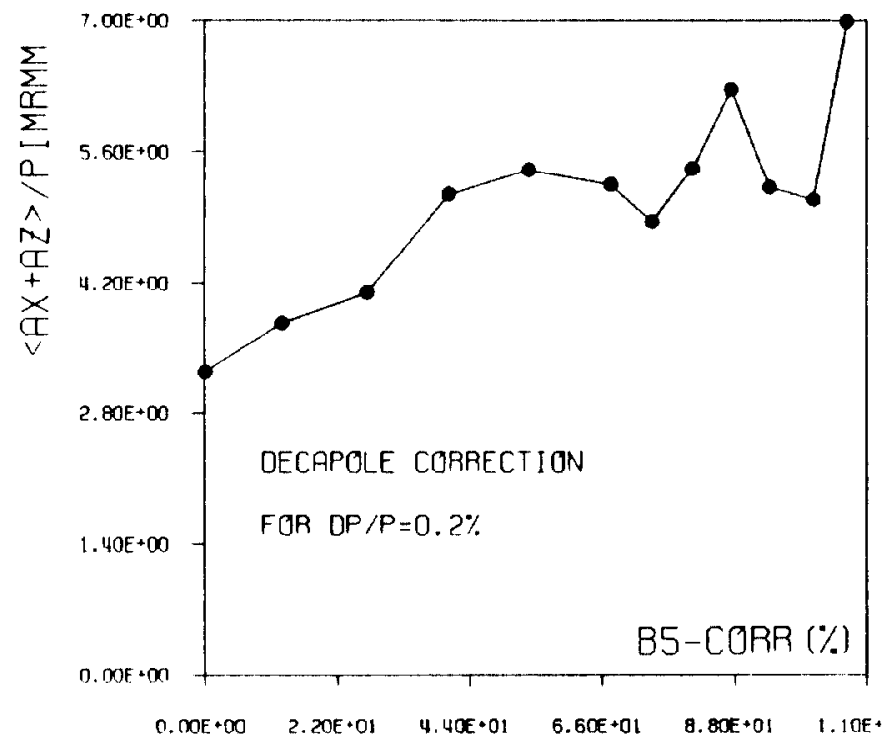

Figure 3: Nonlinear acceptance of the HERA Proton Ring at injection energy taking into account magnetic field errors as measured for each magnet. The dynamic aperture versus decapole corrector strength in units of integrated decapole error is plotted.

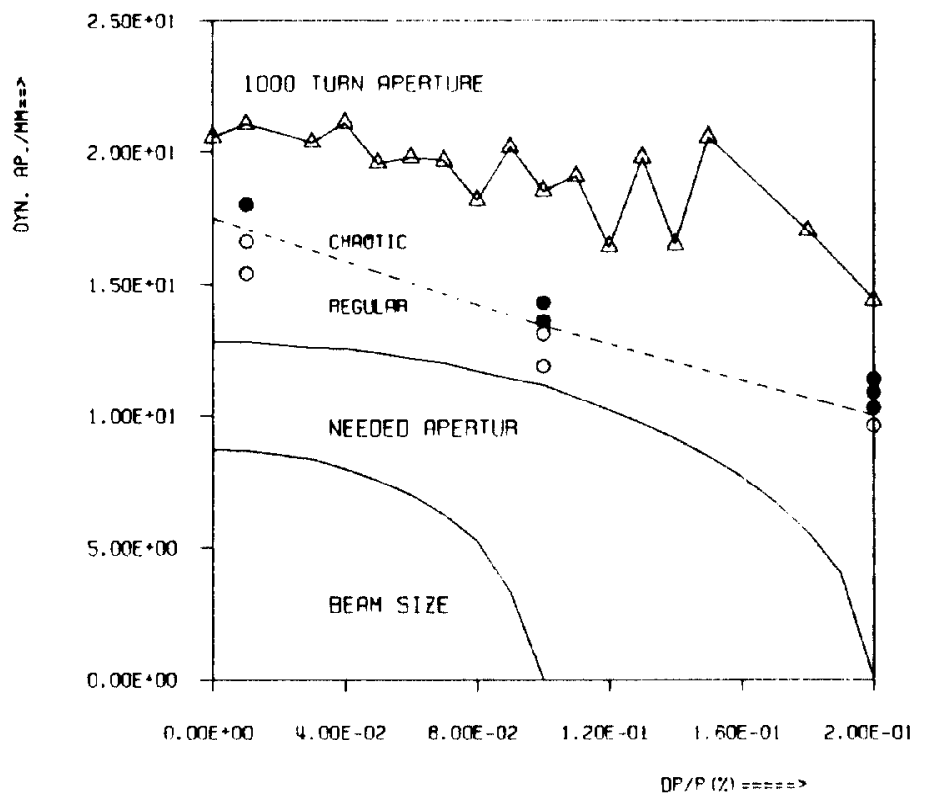

Figure 4: Dynamic Aperture in the HERA proton Ring at injection energy taking into acount magnetic field errors as measured for each magnet. The dynamic aperture versus the amplitude of momentum uscillations is plotted The dashed line represent the estimated dynamic aperture limit. Circles indicate trajectorits with regular behavior, dots are chaotic trajectories. results of field measurement of each individual magnet is summarized in Fig. 4 (see also [8]). Field errors have been taken into account by five thin lenses per half cell. The nonlinear acceptance versus the amplitude of the momentum deviations is plotted. The dashed line divides stable from unstable regions in phase space. The full lines indicates the beam size and the aperture necessary for safe operation [12].

The time dependence of persistent currents causes an operational problem. If the p.c. sextupoles are reinduced afler slow decay at the start of the ramp, a rapid change in chromaticity occurs which has to be tracked by the correction system. From a single particle stability point of view, the chromaticity needs to be kept close to zero in order to avoid strong chaos due to tune modulations by synchrotron oscillations. The possible damage which is caused by uncompensated chromaticity is demonstrated in Fig. 5 which shows the time evolution of the Lyapunov exponent observed in particle tracking for two cases in HERA-p at injection energy. Case (1) has zero chromaticity and oscillation amplitudes well within the dynamic aperture. The motion is clearly regular, thus stable since the Lyapunov exponent approaches zero. In case (2) one starts with the same amplitude but the chromaticity amounts to $\xi_{x}=-16$ and $\xi_{y}=-18$ which corresponds to the effect of $b_{3}=2 \cdot 10^{-4}$. The motion is apparently chaotic, the Lyapunov exponent remains finite.

Time dependent p.c. sextupole fields have also been observed in the TEVATRON, where ther cause emittance blow up and beam loss at the start of the ramp 1. However, once the problem had been recognized. it could easily be handled by programming the sextupole correctors appropriately [14]. This experience is very valuable for HERA. Although the p.c. sextupole of the TEVATRON magnets is with $b_{3}=-4.75 \cdot 10^{-4}$ much smaller than the p.c. sextupole of $b_{3}=-33 \cdot 10^{-3}$ in the HERA dipole. its time dependence is with $\Delta b_{3} \simeq 1 \cdot 10^{-4}$ almost half as strong. Since the TEVATRON transition energy $\gamma_{\tau}$ is only half the HERA $\gamma_{+}$, the effect of time dependent $b_{3}$ on chromaticity is with $\Delta \xi \simeq 20$ comparable for both machines. The spread in p.c. sextupole decay causes a time dependent distribution of nonsystematic sextupole errors around the machine. This spread has the same order of magnitude as the spread in $b_{3}$ due to geometric imperfections. Therefore it is questionable whether the effort of sorting or binning of superconducting magnets according to their nonlinear field properties in order to reduce the harmonic content of these errors in the machine is worthwhile. For the HERA proton ring, the sorting of the dipole magnets according to the geometrical sextupole errors has only a marginal effect on the dynamic aperture at injection field 


\section{HERA-p COMMISSIONING}

In the period of March/April 1991, the technical commissioning of the HERA Proton ring with a $40 \mathrm{GeV}$ proton beam started. The results concerning beam stability are still very preliminary since the tests of technical components of the machine were given priority. $l l$ is interesting however, that a proton beam could be stored in HERA. $p$ with a lifetime of about 30 minutes. The beam optics was found to be in fairly good agreemen with the theory the closed orbit was globally corrected to less than $5 \mathrm{~mm}$ with possible larger deviations at a few positions with faulty monitors. The dipole magnets were excited in a special cycle which avoids strong sextupole components of the injection dipole field 1 see $15 \%$. Chromaticity measurements yielded $b_{3}$ values of 3-4 units of $10^{-4}$. The lifetime is presumably given by a heating process perhaps due to multiple scattering in the straight sections where the vacuum bake out has not yet been performed. Measurements of the lifetime versus the physical aperture however indicate, that nonlinearities may causc an enhancement of diffusion speed. Nevertheless no beam loss could be observed while sweeping slowly across $1 / 5,1 / 6$, and $1 / 7$ integer resonances. $1 / 3$ and $1 / 4$ integer resonances are as expected very strong. Lifetimes of $30 \mathrm{~min}$ were achieved after the chromaticities had been corrected to less than one unit and the coupling resonance had been carefully minimized.

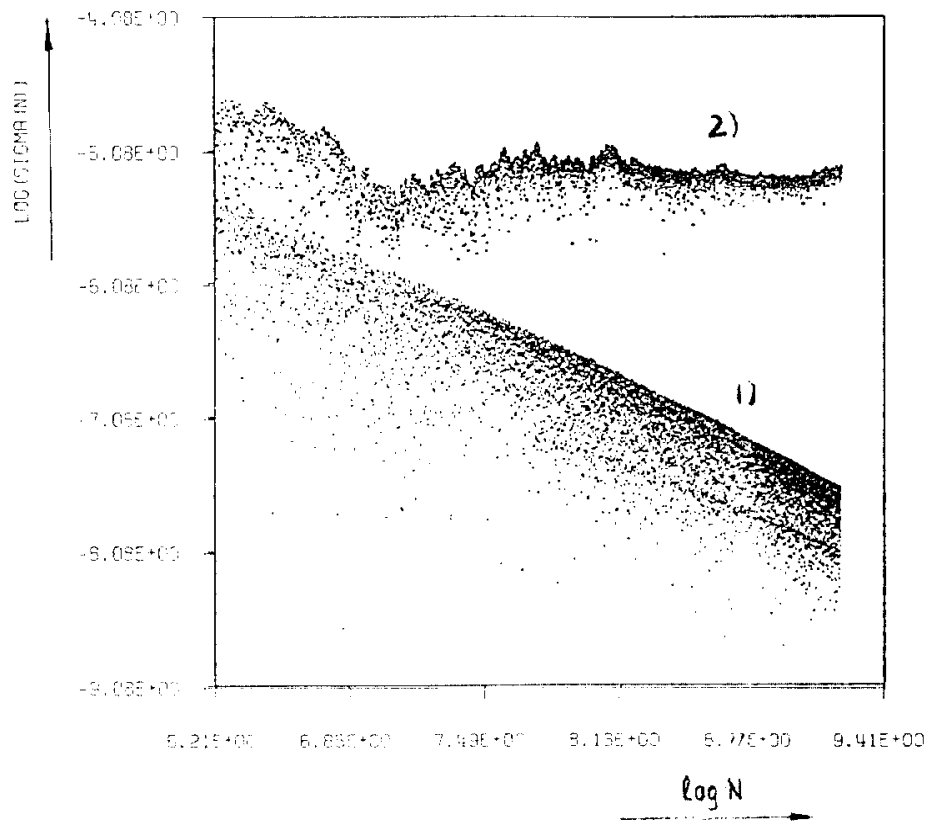

Figure 5: Time evolution of Lyapunor Coefficients for the HERA proton ring taking into account all magnetic field errors as measured. Plotted is the time evolution of the Lvapunov exponent. Curve 1) zero chromaticity, Curve 2) chromaticity $\Delta t_{3}=2 \cdot 10^{-4}$

\section{SUMMARY}

The field quality of the HERA superconducting magnets is remarkably good. Unavoidable strong systematic persistent current sextupolar, decapolar and dodecapolar field errors have to be compensated by compensation coils. Differences between the production lines do not cause problems due to ordered sequences of magnet installations. Simulations of single particle stability of a proton beam in HERA at injection energy promises sufficient aperture for stable synchrobetatron oscillations.

Meanwhile, the HERA proton ring has come into operation. There is no final conclusion yet about the effect of p.c. field errors and whether the correction system is adtquate since the commissioning is still at an early stage. It has. however. been demonstrated that a proton beam can be stored in $\mathrm{HERA}$ at $40 \mathrm{GeV}$ with a reasonable lifetime In the present operation mode of constant momentum of $40 G \in \mathrm{l}$ persistent current field errors do not play a signficant role in the stability of the beam.

\section{References}

[1] D.-A. Finley et al., IEEE PAC. Washington D.C (1989) pp 151-153

[2] H. Brück. R. Meinke, F. Müller. P. Schmüser. DESY 89-041 (1989)

[3] P.W. Anderson, Phys. Rev. Letters 9. 309 (1962)

[4] H. Brück et al. DESY HERA 90-01 (1990)

[5] R. Hanft et al., IEEE Trans. Nucl. Science. Vol. NS 30 Nr. 4 (1983)

[6] G. Benettin L. Galgani, A Giorgilli, J.M Strelcyn Meccanica 15 No 9,21 (1980)

7 F. Schmidt. F. Zimmermann, F. Willeke DESY HERA 91-07 (1991), CERN/SL/51-14(AP)

[8: F. Zimmermann, F. Willeke DESY HERA 91-08 (1991)

19] B.V. Chirikov, Phys. Rep. 52 Nr.5 (1979) pp. 263-379

[10] R. Brinkmann, F. Willeke, DESY HERA 88-08 (1988)

11] A. Wrulich, DESY 84-026 (1984)

12. F. Willeke. DESY IIERA 88-07 (1988)

13 G. Ripken. F. Willeke, DESY 89-07 (1989)

[14 D. A. Herrup et al., IEEE PAC, Chicago (1989) pp. $518-520$

15] P. Schmüser, this conference 